\title{
CLINICOPATHOLOGICAL CONFERENCE
}

\section{An Infant with Interstitial Lung Disease}

\author{
Pankaj C Vaidya $^{1}$, Pandiarajan Vignesh ${ }^{1}$, Kushaljit Singh Sodhi $^{2}$, Meenu Singh $^{1}$ and Uma NahaR ${ }^{3}$ \\ From Departments of ${ }^{1}$ Pediatrics, ${ }^{2}$ Radiology and ${ }^{3}$ Histopathology; Postgraduate Institute of Medical Education and Research, \\ Chandigarh, India. \\ Correspondence to: Dr Uma Nahar, Professor, Department of Histopathology, Postgraduate Institute of Medical Education and \\ Research, Chandigarh 160 012, India.umasaikia@gmail.com
}

\begin{abstract}
Interstitial lung disease in infants, unlike older children and adults, has diverse etiology, including infective, metabolic, autoimmune, genetic, malignant and idiopathic causes. Clinical recognition of the interstitial pattern of lung involvement is important as the etiology and management is entirely different from that of recurrent or chronic lung parenchymal pathologies. We discuss the clinical and pathological findings of an infant with interstitial pneumonia, who succumbed to hospital-acquired sepsis.
\end{abstract}

Keywords: Acute respiratory distress syndrome, Atypical pneumonia, Chronic lung disease.

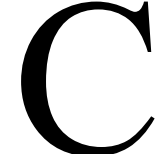
hildhood interstitial lung disease (ChILD) is clinically recognized when there is prolonged history of a non-productive cough, tachypnea more than chest wall retractions, minimal to absent chest auscultatory signs, normal cardiac examination, the presence of clubbing and hypoxemia responsive to oxygen [1]. Etiologies of ChILD are diverse, and corroborative clinical or laboratory features help in guiding focused investigations [2]. We describe the clinicopathological case conference of an infant with interstitial lung disease (ILD), where the various etiologies of ChILD and importance of lung histopathology are discussed.

\section{Clinical Protocol}

History: A one-year-old boy was hospitalized with complaints of fever, cough, difficulty in breathing of one month duration, and intermittent bluish discoloration of lips, palms, and soles, especially on crying. Fever was intermittent, documented up to $104^{\circ} \mathrm{F}$, responding to antipyretics. Cough was non-paroxysmal, associated with difficulty in breathing and feeding disturbance. The child had been previously admitted twice for cough and breathing difficulty; first episode at 5 months of age, and another at 9 months of age. He was treated with intravenous (IV) antibiotics and oxygen; however, the breathing difficulty persisted. He was born by a full term normal delivery at a hospital with a birthweight of $2.5 \mathrm{~kg}$ and had normal neonatal transition. He had been exclusively breastfed until 7 months of age and was on a milk-based diet along with breastfeeds thereafter. He was the only child of the non-consanguineous parents. Father was a smoker and there was no history of contact with pulmonary tuberculosis (TB). The child had been immunized for age, but BCG scar was absent.

Examination: Anthropometric evaluation revealed the weight for age $<3$ rd centile, length for age was at 5 th centile, weight for length was $72 \%$ of expected, which indicated acute-on-chronic malnutrition. The occipitofrontal circumference was at 5 th centile. At admission, heart rate was $160 / \mathrm{min}$, respiratory rate was 80 / min, blood pressure was $90 / 60 \mathrm{~mm} \mathrm{Hg}$, with good normal volume pulses, and normal capillary refill time. Oxygen saturation $\left(\mathrm{SpO}_{2}\right)$ was $50 \%$ on room air, which improved to $97 \%$ on oxygen support using nasal prongs $(5 \mathrm{~L} / \mathrm{min})$. Grade III pan-digital clubbing and anemia were noted. Respiratory system examination showed minimal subcostal retractions, without any crepitations or wheeze on auscultation. Abdominal, cardiovascular and central nervous system examinations were normal.

Investigations: Investigations revealed hemoglobin of 8.4 $\mathrm{g} / \mathrm{dL}$ which dropped significantly to $6.4 \mathrm{~g} / \mathrm{dL}$ during the course of hospital stay, requiring two packed red cell transfusions. There was persistent polymorphonuclear leukocytosis (absolute neutrophil count: 16,000-18,500/ $\mathrm{mm}^{3}$ ) and thrombocytosis $\left(7.51\right.$ lakhs $\left./ \mathrm{mm}^{3}\right)$. Peripheral smear revealed microcytic hypochromic anemia. Alanine transaminase and aspartate transaminase levels were $24 \mathrm{U} / \mathrm{L}$ and $34 \mathrm{U} / \mathrm{L}$, respectively. Arterial blood gases showed persistent respiratory acidosis. Chest radiograph showed bilateral haziness with infiltrates (Fig. 1). Highresolution computerized tomography (HRCT) of the chest revealed features of bilateral diffuse ILD, pulmonary artery hypertension and mediastinal lymphadenopathy. Echocardiogram showed small atrial septal defect with a 


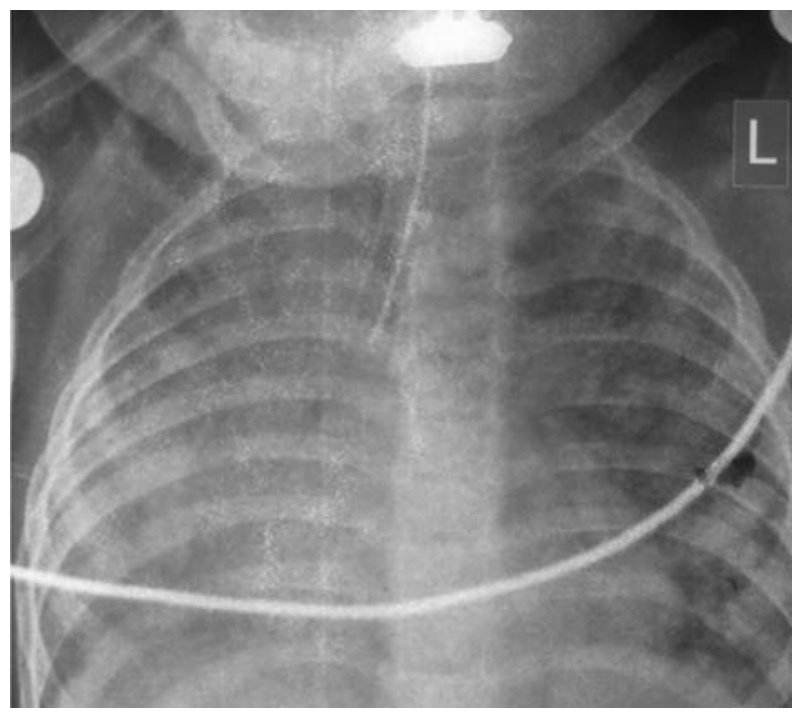

FIG. $1 X$-ray anterio-posterior view of chest showing bilateral interstitial infiltrates and ground glass opacities.

left to right shunt. Work-up for the causes of ChILD is summarized in Table I.

Course and management: Child had a hospital stay of around 2.5 months. At admission, he was started on nasal prongs oxygen ( $5 \mathrm{~L} / \mathrm{min})$ and IV ceftriaxone, amikacin, and cloxacillin. Later azithromycin was added to cover atypical pneumonia and cotrimoxazole for suspected Pneumocystis jirovecii pneumonia (PJP). As the clinical and radiological features suggested an ILD, oral prednisolone was started at a dose of $2 \mathrm{mg} / \mathrm{kg}$ for 14 days followed by tapering over next 4 weeks. The infant responded to steroids initially as his respiratory rate and breathing difficulty decreased though the oxygen requirement persisted. However, on the day 20 of steroid tapering, the child developed marked respiratory distress, fever, lethargy, increasing CRP and features of disseminated intravascular coagulation (DIC) with platelet count of $27,400 / \mathrm{mm}^{3}$. The child was subsequently intubated and given supportive care in the form of platelets and albumin transfusion. The dose of steroids was again hiked up and antibiotics were added. The child also had pre-terminal worsening of liver and renal functions. The endotracheal aspirate culture showed Acinetobacter spp. Child persisted to have severe sepsis with refractory shock and suffered cardiac arrest and could not be revived.

Unit's final diagnosis: The unit's final diagnosis was ChILD with healthcare-associated infection - sepsis with multiorgan dysfunction syndrome (MODS). The cause of death was kept as acute respiratory distress syndrome (ARDS).
Clinical discussion: We have an infant with features of ILD - prolonged history of cough and breathing difficulty, failure to thrive (FTT), tachypnea, hypoxemia which improved with oxygen, pan-digital clubbing, normal cardiac examination, supportive chest radiograph and HRCT features. Child's respiratory status improved with corticosteroids. Another important point to note is that this child had anemia which required packed red blood cell transfusion twice. This case could be approached under two subheadings - etiological approach and the preterminal events.

Index child had an insidious onset diffuse ILD, which encompasses a broad group of diseases that affect the respiratory function of the lung by impairing alveolar gas exchange [2]. As the child was one-year-old, causes like developmental disorders, disorders which are related to systemic diseases, diseases of immunocompromised host, diseases of normal hosts, aspiration syndromes and infections need to be discussed (Table II). ChILD differs from the adult forms of ILDs because here the injury occurs during the development and differentiation of lung and etiology also includes various genetic and metabolic causes [3]. Disorders of lung growth and development are unlikely in our child as the neonatal course was

TABLE I INVESTIGATIONS FOR DIFFUSE INTERSTITIAL LUNG DiSEASE IN THE INDEX CHILD

Infectious diseases work-up

*AFB staining, fungal smears and $P$. jirovecii:

Negative - repeated thrice

*AFB cultures and fungal cultures: Negative

Tuberculin skin testing $0 \mathrm{~mm}$

HIV, CMV serologies: Negative

Immunology work-up

Absolute lymphocyte counts $4,600 / \mathrm{mm}^{3}$

IgG, IgA, IgM values: 692 mg\%, 76 mg\%, 193 mg\%, respectively

Connective tissue disorders/Pulmonary hemosiderosis workup

Urine examination: Albumin- negative, no RBC casts

*Hemosiderin-laden macrophages: Negative

ANA, ANCA: Negative

Other work-up

Sweat chloride levels: $40 \mathrm{mEq} / \mathrm{L}$

Ultrasound abdomen: Normal study

Fundus examination: No cherry red spot

AFB: Acid-fast bacilli; HIV: Human Immunodeficiency Virus; CMV: Cytomegalovirus; Ig: Immunoglobulin; RBCs: Red blood cells; ANA: Antinuclear antibody; ANCA: Anti-neutrophil cytoplasmic antibody; *Gastroic lavage specimens. 
uneventful. Neuroendocrine cell hyperplasia of infancy and pulmonary interstitial glycogenosis also seems unlikely as the presentation would be much earlier in these disorders. Genetic disorders of surfactant production and metabolism could be a possibility; however, surfactant protein B mutations are unlikely as the presentation in those cases would be in early infancy. Whereas pulmonary microlithiasis, vascular, lymphatic disorders, pulmonary infiltrates with eosinophilia could be ruled out because supportive laboratory or radiological evidence were not seen. Human immunodeficiency virus (HIV) and tuberculosis (TB) are important infectious causes that can present with failure to thrive (FTT), ChILD and mediastinal adenopathy $[4,5]$. Lymphoid interstitial pneumonitis is a known feature of HIV. However, HIV serology was negative and multiple gastric lavage samples for acid-fast bacilli staining were not suggestive. TB would still be a strong possibility as it is endemic in India and pediatric TB is usually paucibacillary [6]. Cytomegalovirus (CMV) infection, either acquired by perinatal transmission or in a setting of primary immunodeficiency (PID), could result in persistent interstitial pneumonia. However, there was no accompanying thrombocytopenia or transaminitis, and CMV serology was negative. PJP could also result in interstitial shadows, but symptoms are usually acute and it occurs in a setting of primary or secondary cellular PID [2]. Absolute lymphocyte counts of the child and immunoglobulin profile were normal, thereby ruling out most of the common PIDs.

Cystic fibrosis (CF) is another possibility that could be considered as there is a chronic respiratory illness, malnutrition, FTT and clubbing. However, odd points are normal sweat chloride levels, absence of typical imaging features of chest wall hyperinflation, and absence of pancreatic insufficiency. Pulmonary hemosiderosis could be a likely cause as the child also had microcytic hypochromic anemia requiring packed red cell transfusions. The classic triad consists of iron deficiency anemia, alveolar infiltrates on chest radiograph and hemoptysis which may not be present in all children. However, gastric aspirates for hemosiderin-laden macrophages (HLM) done thrice were negative. Adult type idiopathic ILD can also occur in children and this possibility could not be excluded. Ground glass opacities with sparing of the upper and middle lobes, and cystic changes in lungs, as seen on HRCT chest of the index child, could be seen in non-specific interstitial pneumonitis and lymphocytic interstitial pneumonia. Drug-induced lung diseases, sarcoidosis, and hypersensitivity pneumonitis are unlikely because they are more common in adult age group.
TABLE II Etiologies FOR InTERstitial AND DiffuSe Lung DISEASES OF INFANTS

Diseases common in the infancy age group

Developmental disorders

Congenital alveolar dysplasia

Acinar dysplasia

Alveolar-capillary dysplasia with pulmonary vein misalignment

Growth abnormalities

Neonatal lung diseases- Bronchopulmonary dysplasia (in preterm) and chronic lung disease (term neonates)

Structural lung changes- Trisomy 21

Pulmonary hypoplasia

Lung disease associated with congenital heart disease

Conditions of undefined etiology

Neuroendocrine cell hyperplasia of infancy

Pulmonary interstitial glycogenosis

Surfactant metabolism disorders

Mutations in SPFTB, SPFTC, ABCA3

Diseases that are not specific to infancy age group

Normal host

Infections and post-infectious

Hypersensitivity pneumonia

Aspiration syndromes

Eosinophilic pneumonia

Immunocompromised host

Opportunistic infections (HIV, primary immunodeficiency)

Transplant rejection syndromes

Systemic diseases

Storage disorders- familial lysinuric protein intolerance, Niemann Pick disease

Autoimmune conditions- SLE, JDMS, ANCA-associated vasculitides, sarcoidosis

Langerhans cell histiocytosis

Diseases mimicking ILD

Hypertensive vasculopathy

Lymphatic malformations

Congestive vasculopathy due to cardiac dysfunction or venoocclusive diseases

Unclassified

End stage disease, lung biopsies that are inadequate or nondiagnostic

JDMS: Juvenile dermatomyositis; SLE: Systemic lupus erythematosus; ANCA: Anti-neutrophil cytoplasmic antibody; HIV: Human immunodeficiency virus.

Systemic disorders that could lead to ILD are less likely in the index child as there were predominant lung 
complaints and there was no clinical evidence of other organ system involvement. Langerhans cell histiocytosis (LCH) of the lung may have the clinical presentation of an ILD; however, HRCT usually shows multiple cystic spaces [2]. Index child had predominant ground glass opacities and there was no ear discharge, seborrhea or hepatosplenomegaly. Familial lysinuric protein intolerance is a rare inborn error of metabolism that can result in progressive interstitial pneumonitis; however, there were no other suggestive features like hepatosplenomegaly, sparse brittle hair, or centripetal obesity. Niemann-Pick disease could also have an interstitial lung involvement; however, there is no hepatosplenomegaly in our child [2]. Neurocutaneous syndromes are unlikely because there were no neurocutaneous markers.

Pre-terminally the child had nosocomial sepsis, a tracheal aspirate culture that showed Acinetobacter spp, and shock which was multifactorial (hypoxic, septic and neurogenic). The child also had MODS in the form of acute renal failure (ARF), ARDS and encephalopathy, which ultimately led to brain death. I would like to conclude by saying that it is a ChILD most likely due to: (i) infectious etiology - either TB or CMV and (ii) CF or an idiopathic pulmonary hemosiderosis that cannot be ruled out with associated pulmonary artery hypertension, nosocomial sepsis, and MODS.

\section{Open Forum}

Pediatric pulmonologist: Chest $X$-ray of the child showed diffuse alveolar opacities, with alveoli difficult to differentiate from the interstitium. TB is one disorder that can present with ILD, but none of the investigations were positive. In fact, the child showed a good response to steroids with improvement in oxygen requirement but succumbed to hospital-acquired sepsis. As already discussed, there are so many features not supporting the diagnosis of $\mathrm{CF}$ in this child.

Pediatrician 1: I would like to include primary ciliary dyskinesia as a differential diagnosis here as there are features of early cystic dilation of alveoli, FTT, and recurrent pneumonia. Pulmonary hemosiderosis that can have recurrent pneumonia-like presentation associated with a recurrent drop in hemoglobin is another possibility. Actually, ILD is a broad bag consisting of a variety of disorders hence we really cannot pinpoint from clinical presentation alone.

Pediatric hemato-oncologist: This child did have some interstitial cystic spaces and mediastinal lymph nodes. Hence, the possibility of LCH cannot be still ruled out. Skeletal survey if done could have identified classical punched out lesions.

\section{Pathology Protocol}

A complete autopsy was performed on this one-year-old child. The brain was essentially normal on gross and microscopic examination. There was $300 \mathrm{~mL}$ of serous fluid in both pleural cavities. No excess of fluid was seen in pericardial and peritoneal cavities.

Both the lungs weighed 315 gms, were heavy, firm with patchy pleuritis. There was cobblestone appearance visualized more on the right side. On slicing, the lungs were non-crepitant with a solid appearance. Upper lobes showed microcystic change with honeycombing and intervening scarred areas. Microscopically there was a marked proliferation of type 2 pneumocytes with focal muscularization of alveolar septae. Few foci showed lymphomononuclear infiltrate within interstitium and giant cell transformation (Web Fig. 1). No viral inclusions were seen. An occasional focus of osteoid formation was noted. Other areas revealed fresh and old alveolar hemorrhages with septal fibrosis. Features of pulmonary artery hypertension were present in the form of medial hypertrophy and intimal proliferation. Patchy early bronchopneumonia and pulmonary thromoboembolism was also seen possibly as a result of terminal events (Web Fig. 1).

The heart weighed $78 \mathrm{~g}$ and was normal in shape. All the valves were within normal limits, no vegetations were seen. The right ventricle wall thickness measured $0.4 \mathrm{~cm}$, suggesting hypertrophy. The liver was $350 \mathrm{~g}$ in weight, pale and greasy to feel with exaggerated mottling on the cut surface. Microscopically there was diffuse microvesicular steatosis with sinusoidal dilatation and congestion. Spleen weighed $20 \mathrm{~g}$ with small subcapsular hemorrhages. Kidneys weighed $80 \mathrm{~g}$, appeared swollen and dusky with medullary congestion. The glomeruli showed fibrin thrombi within capillaries. Pigmented casts in the tubules were noted and there were no vascular changes (Web Fig. 2).

Pancreas showed focal acinar dilatation along with features of early acute pancreatitis and terminal fat necrosis. There was dullness of serosal aspect of the entire gastrointestinal tract. The descending colon showed pin head to umbilicated lesions on the mucosa covered with mucus. Peyer's patches appeared prominent. Microscopically the crypts were dilated with a focal pseudomembrane formation. Prominent lymphoid follicles and edema were present in the submucosa.

Final Autopsy diagnosis was Acute interstitial pneumonia (AIP), Pulmonary arterial hypertension with right ventricular hypertrophy, Early bronchopneumonia with pulmonary thrombo-embolism, Passive congestion 
and steatosis of Liver, Disseminated intravascular coagulation of Kidneys, and Ischemic colitis.

\section{Open Forum}

Pediatric pulmonologist: Open lung biopsy is considered to be the gold standard investigation for ILD as it shows the actual pathology and narrows the etiological spectrum. However, many times this would not be feasible for these children as they usually have a severe respiratory failure or an associated infection. Bronchoalveolar lavage could have given some clues especially in identifying infections, or an eosinophilic lung disease.

Pediatrician 1: Surfactant metabolism disorders are likely with this kind of diffuse interstitial lung involvement with the proliferation of type 2 pneumocytes. If lamellar bodies are found, analysis of those structures would have pinpointed the diagnosis. The lungs also showed hemorrhages, HLM, and fibroblast proliferation. This could be well due to ARDS, DIC, and associated pulmonary hypertension. However, if this is extensive, the possibility of primary pulmonary hemosiderosis could be entertained.

Adult pulmonologist: There are lymphoid aggregates and honey-comb like cysts. Moreover, there is only minimal fibroblastic response. Why can't this be a usual interstitial pneumonia (UIP) or a non-HIV related lymphoid interstitial pneumonia (LIP) in this case?

Pathology discussant: There was a florid type 2 pneumocyte proliferation in this case, which is not usually seen with UIP. Lymphoid aggregates are focal and not uniform; hence LIP cannot be labeled here. The presence of fibroblastic foci and widespread inflammatory infiltrates are usually not seen in pulmonary hemorrhage syndromes.

\section{Discussion}

Childhood interstitial lung diseases (ChILD) comprise of a wide spectrum of disorders involving the lung parenchyma and the etiology remains uncertain in majority of cases [2]. The incidence from available studies ranges from 0.5 to 0.8 cases/ 100,000 children per year [1]. Clinical presentation is often non-specific that often contributes to a late clinical diagnosis of this condition. Common clinical features include dyspnea, diffuse infiltrates in the chest radiograph, and impaired gas exchange evidenced by hypoxemia. Acute exacerbations can occur in children with pre-existing ILD due to an infectious trigger, an episode of aspiration or the acceleration of underlying disease process [7].

The index child had a clinical presentation suggestive of ChILD and the pathology revealed the presence of interstitial pneumonitis. No infectious or autoimmune etiologies could be confirmed on the histopathology. AIP is characterized by the presence of acute respiratory failure due to diffuse alveolar damage [8]. Differentiation of AIP from ARDS is difficult on histopathology alone. Prodrome period for ARDS is usually less than a week, whereas in AIP, the prodromal period is usually longer and depends on the etiology. The onset of AIP is usually within 1-3 weeks and a rapid development of respiratory failure may warrant mechanical ventilation in most of the patients [9].

The etiology of AIP is not clearly known; however, treatable infectious causes must be ruled out in a setting of AIP such as bacterial sepsis, viral infections such as influenza, cytomegalovirus, Mycoplasma, and Pneumocystis jirovecii [7]. Rapidly progressive AIP could also occur in connective tissue diseases such as Systemic lupus erythematosus, Juvenile dermatomyositis, Anti-neutrophil cytoplasmic antibodyassociated vasculitis, and Behcet's disease [10]. History, clinical features, and microbiological investigations may reveal potential treatable causes of AIP such as sepsis induced ARDS, systemic connective tissue disorder etc. Sometimes drug-induced or transfusion-induced lung injury can also induce AIP like picture. Patients with cryptogenic fibrosing alveolitis can also have an accelerated presentation similar to the AIP, however, the lung histopathology would reveal features of chronic interstitial pneumonia along with changes of diffuse alveolar damage [11].

Our child did show some improvement following initiation of glucocorticoids. No evidence for autoimmune causes like SLE, Behcet's disease or ANCAassociated vasculitides were identified. The role of bronchoalveolar lavage (BAL) in a case of ChILD is to identify infective causes or developmental anomalies. BAL can also provide evidence for pulmonary alveolar proteinosis, sarcoidosis, or hypersensitivity pneumonitis [12]. BAL could not be done in our child; however, the tracheal aspirate did not identify any potential infective etiology for the ILD. Role of genetic work up for the ILD in infants has been emphasized in the recent literature. Sequencing for $A B C A 3$ and SFTPC gene mutations have been recommended for the work-up for infants with ILD who present after the neonatal period [12]. We acknowledge the non-availability of BAL and genetic work-up in our child.

In general, the management of ChILD involves the empirical use of immunosuppressive therapy. However, in cases of neonates and infants with severe ILD, 
underlying genetic defects must be sorted out for the appropriate genetic counselling. Apart from supportive care, neonates and infants with progressive ILD may also need lung transplantation [13]. Our patient did respond to glucocorticoids and developed relapse while tapering steroids and finally succumbed to hospital acquired sepsis. The histopathology revealed features of AIP. The treatment of AIP is mainly supportive, comprising of supplemental oxygen therapy and invasive or noninvasive mechanical ventilation with positive endexpiratory pressure along with empirical antimicrobials until the bacterial sepsis is reliably ruled out. Early use of corticosteroids has been advocated by many authors and is considered a mainstream therapy in AIP [7]. Immunosuppressive drugs such as intravenous methylprednisolone and cyclophosphamide have also been reported to halt the rapid progression of the disease process in AIP [14]. Other potential experimental therapies that might be useful in AIP include surfactant therapy, inhaled nitric oxide and lung transplantation [8].

Our case highlights one of the rare etiologies for ChILD - AIP, and the etiology is probably idiopathic. Genetic work-up could have thrown light on the surfactant metabolism disorders; however, there was no evidence for pulmonary alveolar proteinosis in the lung histopathology. The diagnosis of ChILD could be made with detailed history, examination, and typical imaging features. Lung biopsy may not be required in all cases to make the diagnosis, and, though genetic tests are needed for confirmation of type of ChILD, treatment should not be delayed while awaiting the results.

Contributors: PCV: prepared the clinical protocol and presented the clinical discussion; KSS: presented the radiology and UN presented the pathology protocol; PCV, PV: prepared the initial draft of the manuscript; KSS,MS, UN: reviewed and revised the manuscript. All authors approved the final draft of the manuscript as submitted; PCV,MS:: were involved in the clinical care of the patient.

Funding: None; Competing interest: None stated.

\section{REFERENCES}

1. Clement A, de Blic J, Epaud R, Galeron L, Nathan N,
Hadchouel A, et al. Management of children with interstitial lung diseases: The difficult issue of acute exacerbations. Eur Respir J. 2016;48:1559-63.

2. Clement A, Nathan N, Epaud R, Fauroux B, Corvol H. Interstitial lung diseases in children. Orphanet J Rare Dis. 2010;5:22.

3. Clement A, Henrion-Caude A, Fauroux B. The pathogenesis of interstitial lung diseases in children. Paediatr Respir Rev. 2004;5:94-7.

4. Shachor Y, Schindler D, Siegal A, Lieberman D, Mikulski $\mathrm{Y}$, Bruderman I. Increased incidence of pulmonary tuberculosis in chronic interstitial lung disease. Thorax. 1989;44:151-3.

5. Zar HJ. Chronic lung disease in human immunodeficiency virus (HIV) infected children. Pediatr Pulmonol. 2008;43:1-10.

6. Kabra SK, Lodha R, Mehta P. 50 years of pediatric pulmonology, progress and future. Indian Pediatr. 2013;50:99-103.

7. Taniguchi H, Kondoh Y. Acute and subacute idiopathic interstitial pneumonias. Respirology. 2016;21:810-20.

8. Bouros D, Nicholson AC, Polychronopoulos V, du Bois RM. Acute interstitial pneumonia. Eur Respir J. 2000;15:412-8.

9. Katzenstein AL, Myers JL, Mazur MT. Acute interstitial pneumonia. A clinicopathologic, ultrastructural, and cell kinetic study. Am J Surg Pathol. 1986;10:256-67.

10. Suda T, Kaida Y, Nakamura Y, Enomoto N, Fujisawa T, Imokawa $\mathrm{S}$, et al. Acute exacerbation of interstitial pneumonia associated with collagen vascular diseases. Respir Med. 2009;103:846-53.

11. Akira M. Computed tomography and pathologic findings in fulminant forms of idiopathic interstitial pneumonia. $\mathrm{J}$ Thorac Imaging. 1999;14:76-84.

12. Wambach JA, Young LR. New clinical practice guidelines on the classification, evaluation and management of childhood interstitial lung disease in infants: what do they mean? Expert Rev Respir Med. 2014;8:653-5.

13. Kurland G, Deterding RR, Hagood JS, Young LR, Brody AS, Castile RG, et al. An official American Thoracic Society Clinical Practice Guideline: Classification, Evaluation, and Management of Childhood Interstitial Lung Disease in Infancy. Am J Respir Crit Care Med. 2013;188:376-94.

14. Pratt DS, Schwartz MI, May JJ, Dreisin RB. Rapidly fatal pulmonary fibrosis: The accelerated variant of interstitial pneumonitis. Thorax. 1979;34:587-93. 